\title{
Philosophiques
}

\section{Luc Brisson. Platon 1958-1975. Lustrum 20 (1977). Göttingen, Vandenhoeck \& Ruprecht, 1979, 304 p.}

\section{Georges Leroux}

Volume 8, numéro 1, avril 1981

URI : https://id.erudit.org/iderudit/203158ar

DOI : https://doi.org/10.7202/203158ar

Aller au sommaire du numéro

Éditeur(s)

Société de philosophie du Québec

ISSN

0316-2923 (imprimé)

1492-1391 (numérique)

Découvrir la revue

Citer ce compte rendu

Leroux, G. (1981). Compte rendu de [Luc Brisson. Platon 1958-1975. Lustrum 20 (1977). Göttingen, Vandenhoeck \& Ruprecht, 1979, 304 p.] Philosophiques, 8(1),

197-199. https://doi.org/10.7202/203158ar d'utilisation que vous pouvez consulter en ligne.

https://apropos.erudit.org/fr/usagers/politique-dutilisation/ 


\section{COMPTES RENDUS}

Luc BRISSON. Platon 1958-1975. Lustrum 20 (1977). Göttingen, Vandenhoeck \& Ruprecht, 1979. 304 p.

La tâche que s'est assignée Luc Brisson en entreprenant de poursuivre la bibliographie de $\mathrm{H}$. Cherniss, publiée dans deux volumes antérieurs de Lustrum (4[1959] et S[1960]), était gigantesque. On peut dire qu'elle correspond à un niveau ultime de l'entreprise bibliographique, après quoi la recension de la production académique sur Platon, aussi bien philologique que philosophique, devient impensable. Une bibliographie peut certes se limiter à n'être qu'un catalogue de références, mais aussitôt qu'elle se propose d'analyser les items répertoriés pour les classifier et les mettre en rapport les uns avec les autres, elle doit affronter l'obstacle de la quantité. Comme le dit justement Brisson, la production contemporaine fait de cet obstacle un «écran» qu'il faut à tout prix traverser si on ne veut pas perdre de vue le profil fondamental de la recherche platonicienne.

La période 1958-1975 regroupe sans doute autant de contributions aux études platoniciennes que toutes les époques antérieures mises ensemble. La première époque s'étend des premiers commentateurs de Platon jusque vers 1700, c'est-à-dire jusqu'au début de l'érudition historique et philologique. Elle se caractérise surtout par l'apparition de commentaires qui sont tout à la fois des reprises philosophiques de Platon, comme par exemple chez les grands néoplatoniciens ou chez Marsile Ficin, et des travaux critiques, comme chez Olympiodore. La deuxième époque va de 1700 à la fin du dix-neuvième siècle; elle recoupe le développement de la philologie et se termine avec la mise en place des instruments modernes de la bibliographie courante. Pour l'essentiel, cette production a été recensée dans les bibliographies de Engelmann ${ }^{1}$ et de Klussman ${ }^{2}$, qui furent continuées par la publication de la Bibliothera Pbilologica Classica, à partir de 1874 jusqu'en 1938. La période moderne s'ouvre avec la

1. Wilhelm ENGELMANN. Bibliotbeca Scriptorum classicorum. 8. Auflage, umfassend die Literatur von 1700-1878, neu bearb. von E. Preuss, Leipzig, 1880-82.

2. Rudolf KLUSSMANN. Bibliotbeca Siriptorum classicorum et graecorum et latinorum. Die Literatur von 1878 bis 1896 einschliesslich umfassend, Leipzig, 1904. 
publication de l'Année philologique, précédée des synthèses de Scarlatti ${ }^{3}$ et de Marouzeau ${ }^{4}$.

Jusque-là, il n'était point besoin encore d'une bibliographie platonicienne spécialisée; il était encore possible de se retrouver dans les recherches publiées, dont la partie la plus considérable venait d'Allemagne. La parution, en 1959-60, de la bibliographie de Cherniss correspond à l'explosion de la recherche américaine. En deux tomes, cette bibliographie comprend 2025 titres. Cet ordre de grandeur permettait à l'auteur de commenter plusieurs titres et de fournir des références croisées (cross-index) extraordinairement utiles. Remarquons cependant que la période recensée par Cherniss était assez restreinte: $1950-1957$ !

Prenant la suite de Cherniss, Luc Brisson recense 3326 titres pour une période (1958-75) deux fois plus étendue. Sa bibliographie est nécessairement sélective; elle écarte notamment les chapitres d'ouvrages qui ne sont pas entièrement consacrés à Platon. Ce choix s'imposait en raison du volume de la production. La bibliographie de Brisson ne pouvait pas non plus être aussi fortement analytique que celle de Cherniss, encore une fois compte tenu de la quantité. Que faire avec plus de trois mille titres qui soit autre chose qu'une liste brute?

La solution adoptée par Brisson est ingénieuse, mais d'utilisation complexe. Il s'agit de confier à un index analytique sophistiqué la tâche de fournir à l'urilisateur l'information nécessaire en l'absence de descripteurs pour les items séparés. Cet index permet d'abord d'exclure (p. 256) nombre de travaux secondaires et redondants. Il identifie ensuite les travaux qui ont une importance sur le plan bibliographique. Puis il classifie, à l'aide de numéros d'item, tous les titres répertoriés selon des catégories qui reprennent pour l'essentiel le modèle de Cherniss. On retrouvera notamment dans Brisson l'indispensable classification des études selon les passages du corpus platonicien (p. 264-87). Pour ce qui est de l'index des thèmes ou concepts, Brisson a eu la bonne idée de fournir de courtes annexes qui constituent à vrai dire des synthèses lapidaires des problèmes qui préoccupent les platonisants d'aujourd'hui (p. 288-97). Malgré sa trop grande brièveté, si on la compare à l'effort de Cherniss, cette section est absolument remarquable de précision et de clarté. Enfin, Brisson fournit un lexique qui comprend deux indices, l'un onomastique et l'autre terminologique, des termes grecs.

Si on compare l'ouvrage de Luc Brisson au modèle fourni par son illustre prédécesseur, on ne peut que s'étonner du peu de conséquence de l'absence des notices. À vrai dire, Brisson nous donne par le moyen de son index tout le matériel nécessaire pour s'orienter dans la littérature et construire soi-même son parcours de recherche. La seule chose qu'il ne donne pas, - mais les

3. Lambrino SCARLATTI. Bibliographie de l'Antiquité classique. 1896-1914. Paris, Les BellesLettres, 1951.

4. Jules MAROUZEAU. Dix années de bibliograpbie classique. Bibliographie critique et analytique de l'Antiquité gréco-latine pour la période 1914-1924. Paris, Les Belles-Lettres, 1927-28. 2 Vol. 
manquera-t-on vraiment? - ce sont les jugements, les opinions de Cherniss qui évaluait tous les travaux à l'aune de son interprétation, au demeurant rigide, du platonisme. Peut-être Brisson, qui peut être considéré comme un disciple de Cherniss, a-t-il pensé néanmoins qu'il faut réserver à des travaux autres que les bibliographies la tâche de discuter de Platon? Je ne suis pas loin de croire qu'il a eu raison ${ }^{5}$.

Georges Leroux

Université du Québec à Montréal 